\title{
Autoantibodies against zinc transporter 8 are related to age and metabolic state in patients with newly diagnosed autoimmune diabetes
}

\author{
Elżbieta Niechciał ${ }^{1}$ (1) - Anita Rogowicz-Frontczak ${ }^{2} \cdot$ Stanisław Piłaciński ${ }^{2} \cdot$ Marta Fichna $^{3,4} \cdot$ Bogda Skowrońska $^{1}$. \\ Piotr Fichna ${ }^{1}$. Dorota Zozulińska-Ziółkiewicz ${ }^{2}$
}

Received: 2 October 2017 / Accepted: 19 December 2017 / Published online: 11 January 2018

(c) The Author(s) 2018. This article is an open access publication

\begin{abstract}
Aims To assess the prevalence of ZnT8-ab and its correlation to other autoimmune markers and diabetic ketoacidosis occurrence in children and adults with T1DM onset.

Methods The study included 367 patients (218 children; 149 adults) at the T1DM onset. Selected diabetes-related autoantibodies such as GAD-ab, IA2-ab, ZnT8-ab were tested before the initiation of insulin therapy. Diabetic ketoacidosis was defined as glucose concentration $>13.9 \mathrm{mmol} / \mathrm{l}, \mathrm{pH}<7.30$, concentration of $\mathrm{HCO}_{3}<15 \mathrm{mmol} / \mathrm{l}$, presence of ketone bodies in the blood and urine.

Results The autoantibodies pattern differs in both study groups. Children were mostly positive for two (37.8\%) and three (49.5\%) autoantibodies, whereas adults for one (32.2\%) and two (30.7\%). The most frequently detected autoantibodies in youth were ZnT8-ab (81.1\%) and IA2-ab (80.7\%), while in adults GAD-ab (74.8\%). ZnT8-ab $(p<0.0001)$ titers were significantly higher in children, but adults had higher titer of GAD-ab $(p<0.0001)$ and IA2-ab $(p<0.0001)$. Children developed more frequently diabetic ketoacidosis $(28.4$ vs. $10.7 \%, p=0.0002)$. ZnT8-ab $(p=0.002)$ and IA2-ab $(p=0.008)$ were reported mostly in individuals with ketoacidosis. A correlation between the number of positive antibodies and the severity of ketoacidosis was observed $\left(R_{\mathrm{s}}-0.129 p=0.014\right)$. ZnT8-ab were associated with a greater risk of ketoacidosis independent of gender, age group and the autoantibodies number [OR $=2.44$ (95\% CI 1.0-5.94), $p=0.04$ ].

Conclusions Children are at greater risk of ketoacidosis at the diagnosis of diabetes. ZnT8-ab and IA2-ab are commonly detected in children, while adults have frequently higher titer of GAD-ab. ZnT8-ab are associated with more acute diabetes onset.
\end{abstract}

Keywords Childhood type 1 diabetes $\cdot$ Adult type 1 diabetes $\cdot$ Autoimmune diabetes diagnosis $\cdot$ Autoimmunity $\cdot$ Diabetesrelated autoantibodies · Autoantibodies to zinc transporter-8

Parts of this study were presented in abstract form at the 52nd Annual Meeting of the European Associations for the Study of Diabetes, Munich, Germany, 13-16 September 2016.

Managed by Antonio Secchi.

Electronic supplementary material The online version of this article (https://doi.org/10.1007/s00592-017-1091-x) contains supplementary material, which is available to authorized users.

Elżbieta Niechciał

e.niechcial@gmail.com

Extended author information available on the last page of the article

$\begin{array}{ll}\text { Abbreviations } \\ \text { T1DM } & \text { Type 1 diabetes mellitus } \\ \text { DKA } & \text { Diabetes ketoacidosis } \\ \text { GAD-ab } & \text { Glutamic acid decarboxylase autoantibodies } \\ \text { IA2-ab } & \text { Protein tyrosine phosphatase autoantibodies } \\ \text { IAA } & \text { Insulin autoantibodies } \\ \text { ICA } & \text { Islet-cell antibodies } \\ \text { LADA } & \text { Latent autoimmune diabetes of adults } \\ \text { ZnT8-ab } & \text { Autoantibodies to zinc transporter- } 8 \\ \text { HbA1c } & \text { Glycated hemoglobin }\end{array}$

Abbreviations

T1DM Type 1 diabetes mellitus

DKA Diabetes ketoacidosis

GAD-ab Glutamic acid decarboxylase autoantibodies

IA2-ab Protein tyrosine phosphatase autoantibodies

IAA Insulin autoantibodies

ICA Islet-cell antibodies

LADA Latent autoimmune diabetes of adult

HbA1c Glycated hemoglobin 


\section{Introduction}

Type 1 diabetes (T1DM) results from immune dysregulation in which the immune response is specifically directed against pancreatic $\beta$-cells. Despite numerous investigations on T1DM origin, the mechanism of this process remains unclear and T1DM is considered as a multifactorial disease. To this point, no single factor inducing the destructive process has been identified. Genetic susceptibility and many environmental agents, including gluten containing food, play a role in disease origin [1-6].

T1DM is mostly diagnosed in children, but it can emerge in adult individuals as well [7]. Diabetic ketoacidosis (DKA) can be a first manifestation of T1DM at any age. Some individuals may retain sufficient residual $\beta$-cells function to prevent DKA for many years. The loss of the pancreatic islet $\beta$-cells mass seems to be more rapid in children than in adults [8].

The presence of autoantibodies is useful for diagnostic support of T1DM. These comprise islet-cell antibodies (ICA), glutamic acid decarboxylase autoantibodies (GAD-ab), protein tyrosine phosphatase autoantibodies (IA2-ab) and insulin autoantibodies (IAA). The majority of patients at T1DM onset are positive for at least one of above-mentioned antibodies [9]. GAD-ab is the most commonly detected autoantibody, found in $50-80 \%$ individuals $[10,11]$. While IAA occurs in very young children and displays a strong inverse correlation with patient's age at the time of T1DM diagnosis [12, 13], IAA is found in $40-70 \%$ children at diagnosis $[4,6]$. IA2-ab is reported in $32-75 \%$ patients at T1DM onset $[14,15]$. GAD-ab and ICA are also significant in latent autoimmune diabetes of adults (LADA) diagnosis $[16,17]$.

Moreover, diabetes-related autoantibodies might be used as progression markers to T1DM. Velluzii et al. [18] reported that throughout the 10-year study period, the risk of having T1DM was 55.3 times higher in children with any single positive autoantibody and rose by 14.5 times in individuals with any double autoantibodies. Kohler et al. observed a positive association between the time to development of T1DM and the seroconversion occurrence. In detail, a positive association was seen up to 54 months for IAA, between 6 and 36 months for IA2-ab and up to 18 months for GADA-ab. [19]

Recently, ZnT8-ab have been identified as a novel target of T1DM autoimmunity. ZnT8 is a 369 -amino acid transmembrane protein, encoded by the SLC $30 A 8$ gene at the chromosome 8q14.11 [20, 21]. ZnT8-ab are found in 66-80\% Caucasian patients at disease diagnosis. Additionally, based on the study performed by Wenzlau et al., they could be detectable in $26 \%$ individuals with T1DM previously classified as autoantibody negative. Therefore, the autoimmunity detection rate could increase up to $98 \%$ if the measurement of ZnT8-ab was added (20). Nevertheless, ZnT8-ab prevalence varies in different population. For example, ZnT8-ab were present only in $28 \%$ of the Japanese patients at T1DM onset [22].

Although, ZnT8-ab are reported in the majority of T1DM patients prior to and at clinical diagnosis, the relationship between the presence of ZnT8-ab and severity of the initial clinical manifestation remains ambiguous. This study aims to assess and compare the prevalence of ZnT8-ab and its correlation to other autoimmune markers and DKA at the presentation of T1DM in children and adults.

\section{Methods}

\section{Patients}

We studied the prevalence of GAD-ab, IA2-ab and ZnT8-ab in children and adults with newly diagnosed T1DM between 2010 and 2014. Moreover, IAA were measured in pediatric group, and ICA testing was performed in all patients with adult onset of diabetes. All participants were an ethnically homogenous population from Poland. The cohort comprised 367 European Caucasian patients, including: 218 children [girls: 95; boys: 123; median age: 9 years (interquartile range: $6-13$ years)] and 149 adults [women: 78; men: 71 ; median age: 34 years (interquartile range: $27-43$ ) years)], admitted to the Department of Pediatric Diabetes and Obesity, and to the Department of Internal Medicine and Diabetology of the Poznan University of Medical Sciences. The study was approved by the Poznan University of Medical Sciences Ethics Committee (decisions No 1623/05, No 739/09 and No 960/12). Informed consent was obtained from all individual participants included in the study.

\section{Diabetes diagnosis}

Diabetes was diagnosed based upon WHO criteria, including fasting plasma glucose $\geq 7.0 \mathrm{mmol} / \mathrm{l}, 2$-h postprandial plasma glucose $\geq 11.1 \mathrm{mmol} / 1$, during an oral glucose tolerance test, classic symptoms of hyperglycemia or hyperglycemic crisis, as well as random plasma glucose concentration of $\geq 11.1 \mathrm{mmol} / \mathrm{l}$. The first day of insulin administration was at T1DM diagnosis.

\section{Clinical and laboratory data}

Standard laboratory tests, including blood glucose, capillary blood gases and blood or urinary ketones, were performed in every patient. DKA was defined as blood glucose concentration $>13.9 \mathrm{mmol} / 1$, blood $\mathrm{pH}<7.30$, concentration of $\mathrm{HCO}_{3}<15 \mathrm{mmol} / \mathrm{l}$, detection of ketones in the urine or 
elevated ketones in the serum, anion gap $>12$. It was considered as mild, moderate and severe if $\mathrm{pH}$ was $<7.3 \geq 7.2$, $<7.2 \geq 7.1$ and $<7.1$, respectively. Level of C-peptide was assessed by radioimmunoassay [C-PEP II-RIA-CT, DIA source Immunoassay, S.A, Belgium]. Glycated hemoglobin (HbA1c) value was evaluated by Chemiluminescent Microparticlr Immunoassey [HbA1C ARCHITECT System, Abbott Laboratories, U.S.A.]. To confirm autoimmune diabetes origin, typical autoantibodies were tested. The blood samples were collected on the day of diagnosis, before an initial insulin therapy, centrifuged to obtain serum and frozen until analyzed. GAD-ab and IA2-ab were measured by the RIA test kits, GAD-ab assay (positivity: $>1 \mathrm{U} / \mathrm{ml}$ ) and IA-2-ab assay (positivity: $>1 \mathrm{U} / \mathrm{ml}$ ), respectively [GAD and IA2 RIA. EUROIMMUN, Germany]. Level of ZnT8-ab was determined by the enzyme-linked immunosorbent assay [ElisaRSR ZnT8 $\mathrm{Ab}^{\mathrm{TM}}$, UK]. The upper limit of the normal range was $15 \mathrm{U} / \mathrm{mL}$, according to the manufacturer's recommendation. Although IAA were checked in children, these antibodies are less likely to be present in older individuals and hence were not screened in adults and are not considered in the study.

\section{Statistical analysis}

The results are displayed as means and standard deviations $( \pm \mathrm{SD})$, medians and interquartile ranges (IQR) or as numbers and percentages (\%). Normality of the data distributions was tested using D'Agostino-Pearson test. We compared groups with different profiles of diabetes-associated autoantibodies using Student's $t$ test or Mann-Whitney $U$ test for continuous variables and Fisher's exact test for categorical variables. We calculated Pearson's correlation coefficients to determine the association between selected continuous variables and the titer of diabetes-related autoantibodies.

We performed all tests at a significance level of 0.05 (two sided). Statistical analyses were carried out using Statistica version 10 (StatSoft Inc., Tulsa, OK, USA) and MedCalc version 17.5.3 (MedCalc Statistical Software, Ostend, Belgium).

\section{Results}

Laboratory tests revealed that children demonstrated higher glucose concentration $(24.8 \pm 10.6$ vs. $18.8 \pm 8.1 \mathrm{mmol} / \mathrm{l}$, $p=0.0017)$ and lower C-peptide level $(0.4 \pm 0.2$ vs. $1.1 \pm 0.7 \mathrm{pmol} / \mathrm{ml}, p<0.0001)$ compared to adults. Accordingly, children were more frequently diagnosed with DKA (28.4 vs. $10.7 \%, p=0.0002$ ) (Table 1 ).

Two hundred and seven (95\%) children and one hundred twenty-one (81.2\%) adults were classified as at least one of three autoantibody positive at T1DM onset. The occurrence of multiple autoantibodies differed significantly between children and adults. The appearance of a unique autoantibody was rare in children, concerning only $7.8 \%$ individuals. Commonly, two $(37.8 \%)$ or three $(49.5 \%)$ diabetes-associated autoantibodies were detectable in children $(p<0.0001)$ compared to adults. Conversely, adults mostly presented with just one $(32.2 \%)$ or two $(30.7 \%)$ of the assessed antibodies. Only $18.1 \%$ adults displayed three positive autoantibodies (Table 1).

Altogether 65 (17 children and 48 adults) of the 367 patients at T1DM onset were positive for just one autoantibody. Isolated GAD-ab was most frequently found in adults (75.4\%). In children, the occurrence of all three of the assessed antibodies was similar. One hundred and twentyeight patients ( 82 children and 46 adults) were positive for two autoantibodies. IA2-ab and ZnT8-ab were the most frequent couple detected in children $(53.6 \%)$ compared to adults $(4.8 \%)(p<0.0001)$. In adults, mostly GAD-ab with IA2-ab (53.6\%) and GAD-ab with ZnT8-ab (41.4\%) combinations were observed (Figs. 1, 2).

Both study groups demonstrated diverse autoantibodies pattern. In particular, $177(81.1 \%)$ children were positive for ZnT8-ab, 176 children (80.7\%) for IA2-ab and $152(69.7 \%)$ for GAD-ab. IAA were tested exclusively in children and found in 128 individuals (58.7\%) (girls: 58; boys: 42), in $30(24 \%)$ children aged $0-4$ years, 45 (35\%) children aged 5-9 years, $40(31 \%)$ aged 10-14 years and $13(10 \%)$ patients aged $15-18$ years.

On the contrary, only 52 adults $(34.8 \%)$ were positive for ZnT8-ab, 65 (44.0\%) for IA2-ab and 111 (74.8\%) for GAD$\mathrm{ab}$, while 105 adult patients tested ICA positive (70.5\%). Therefore, the presence of ZnT8-ab (81.1 vs. $34.8 \%$, $p<0.0001)$ and IA2-ab (80.7 vs. 44\%, $p<0.0001)$ was more common in children than in adults. Moreover, significantly higher titers of ZnT8-ab [407.7 (35.5-524.5) vs. 6.6 (0.0-157.9) U/ml, $p<0.0001]$ were found among pediatric population. In contrast, GAD-ab titers [3.9 (0.5-17.7) vs. $105.4(8.9-545.8) \mathrm{U} / \mathrm{ml}, p<0.0001]$ and IA2-ab titers [6.5 (1.5-19.3) vs. 17.8 (7.0-111.7) U/ml, $p<0.0001]$ were higher in adults compared to children (Table 1).

We found a positive correlation between age and the titer of GAD-ab $\left(R_{\mathrm{s}}-0.296 p<0.001\right)$ and a negative with the titer of ZnT8-ab $\left(R_{\mathrm{s}}-0.314 p<0.001\right)$ in both studied groups, while lower level of IA2-ab was correlated with increasing age in adults $\left(R_{\mathrm{s}}-0.339 p<0.001\right)$.

Seventy-eight new T1DM cases were complicated by DKA (mild: $n=23$, moderate: $n=36$, severe: $n=19$ ). DKA was mainly observed in children (79.5\%). Of the IAA positives children, 37 developed DKA, and this was moderate in 16, mild in 11 and severe in 11 . ZnT8-ab (79.4 vs. $57.7 \%, p=0.002)$ and IA2-ab (78.2 vs. $61.2 \%$, $p=0.008)$ were detected more frequently in DKA group. Interestingly, the titers of IA2-ab [6.0 (2.5-24.2) vs. 11.0 
Table 1 Clinical features and autoantibodies spectrum in children and adults with newly diagnosed type 1 diabetes

\begin{tabular}{|c|c|c|c|}
\hline Variable & Children $(n=218)$ & Adults $(n=149)$ & $p$ value \\
\hline \multicolumn{4}{|l|}{ Clinical characteristics } \\
\hline Age (year) [IQR] & $9.0(6.0-13.0)$ & $34.0(27.0-43.0)$ & NA \\
\hline $\operatorname{Sex}(\mathrm{F}: \mathrm{M})$ & $95: 123$ & $78: 71$ & NS \\
\hline DKA $(\%)$ & 28.4 & 10.7 & $p=0.0002$ \\
\hline $\mathrm{NGSP} \mathrm{HbA}_{1 \mathrm{C}}(\%)[\mathrm{mmol} / \mathrm{mol}]$ & $11.0 \pm 2.1[97 \pm 18.5]$ & $10.9 \pm 2.7[96 \pm 23.8]$ & NS \\
\hline Glycemia at admission (mmol/l) & $24.8 \pm 10.6$ & $18.8 \pm 8.1$ & $p=0.0017$ \\
\hline C-peptide (pmol/l) & $0.4 \pm 0.2$ & $1.1 \pm 0.7$ & $p<0.0001$ \\
\hline \multicolumn{4}{|l|}{ Autoantibodies } \\
\hline Positive GAD-ab (\%) & 69.7 & 74.8 & NS \\
\hline GAD-ab titer (U/ml) & $3.9(0.5-17.7)$ & $105.4(8.9-545.8)$ & $p<0.0001$ \\
\hline Positive IA2-ab (\%) & 80.7 & 44.0 & $p<0.0001$ \\
\hline IA2-ab titer (U/ml) & $6.5(1.5-19.3)$ & $17.8(7.0-111.7)$ & $p<0.0001$ \\
\hline Positive ZnT8-ab (\%) & 81.1 & 34.8 & $p<0.0001$ \\
\hline ZnT8-ab titer $(\mathrm{U} / \mathrm{ml})$ & $407.7(35.5-524.5)$ & $6.6(0.0-157.9)$ & $p<0.0001$ \\
\hline \multicolumn{4}{|l|}{ Number of positive autoantibodies } \\
\hline 1 antibody $(\%)$ & 7.8 & 32.2 & $p<0.0001$ \\
\hline 2 antibodies (\%) & 37.8 & 30.7 & NS \\
\hline 3 antibodies (\%) & 49.5 & 18.1 & $p<0.0001$ \\
\hline \multicolumn{4}{|l|}{ Individuals positive for single antibody } \\
\hline GAD-ab (\%) & 35.3 & 75.4 & $p=0.013$ \\
\hline IA2-ab (\%) & 29.4 & 12.1 & NS \\
\hline ZnT8-ab (\%) & 35.3 & 12.5 & NS \\
\hline \multicolumn{4}{|l|}{ Individuals positive for two antibodies } \\
\hline GAD-ab + IA2-ab (\%) & 23.2 & 53.6 & $p=0.0011$ \\
\hline GAD-ab + ZnT8-ab (\%) & 23.2 & 41.5 & NS \\
\hline $\mathrm{ZnT} 8-\mathrm{ab}(\%)+\mathrm{IA} 2-\mathrm{ab}(\%)$ & 53.6 & 4.9 & $p<0.0001$ \\
\hline
\end{tabular}

Data are expressed as means $( \pm \mathrm{SD})$ or medians unless otherwise stated. C-peptide reference range: 0.59 $1.54 \mathrm{pmol} / \mathrm{ml}$

$I Q R$ interquartile range, $N A$ non-assessed, $N S$ non-significant

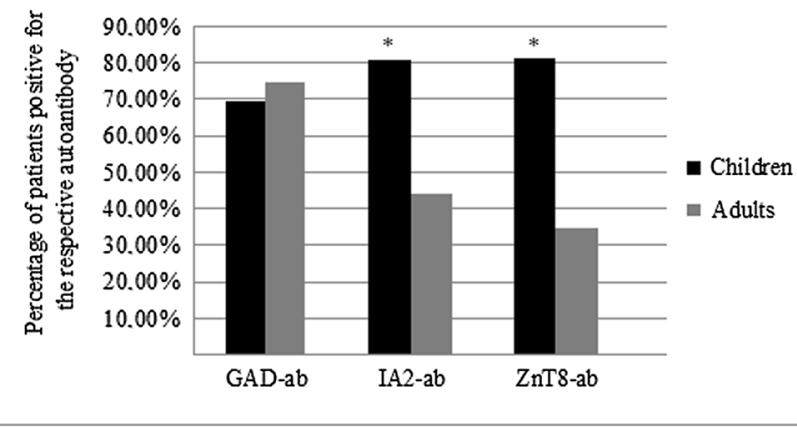

*IA2-ab and ZnTS-ab occurred signific antly often in children $(p<0.0001)$

Fig. 1 Autoantibodies spectrum in children and adults with newly diagnosed T1DM

(2.7-24.4) U/ml, $p<0.0001]$ and GAD-ab [4.7 (0.4-34.5) vs. $9.9(2.2-105.4) \mathrm{U} / \mathrm{ml}, p<0.00001]$ were lower in individuals with DKA (Table 2). There was a significant correlation between the number of positive antibodies and the severity of DKA $\left(R_{\mathrm{s}} 0.129 p=0.014\right)$. In multiple regression analysis, the presence of positive ZnT8-ab was associated with higher risk of DKA independent of gender, agegroup (children and adults) and the number of autoantibodies (0-3) [OR $=2.44$ (95\% CI 1.0-5.94), $p=0.04$ ].

\section{Discussion}

We confirmed that children had more severe symptoms with lower $\beta$-cell function and more frequent DKA at disease presentation. Moreover, children and adults display diverse autoantibody patterns and severity of the autoimmune aggression, as estimated by the number of autoantibody found in both groups. These findings suggest that despite plausible influence of similar environmental factors, the intensity of the autoimmune attack may vary according to age. 

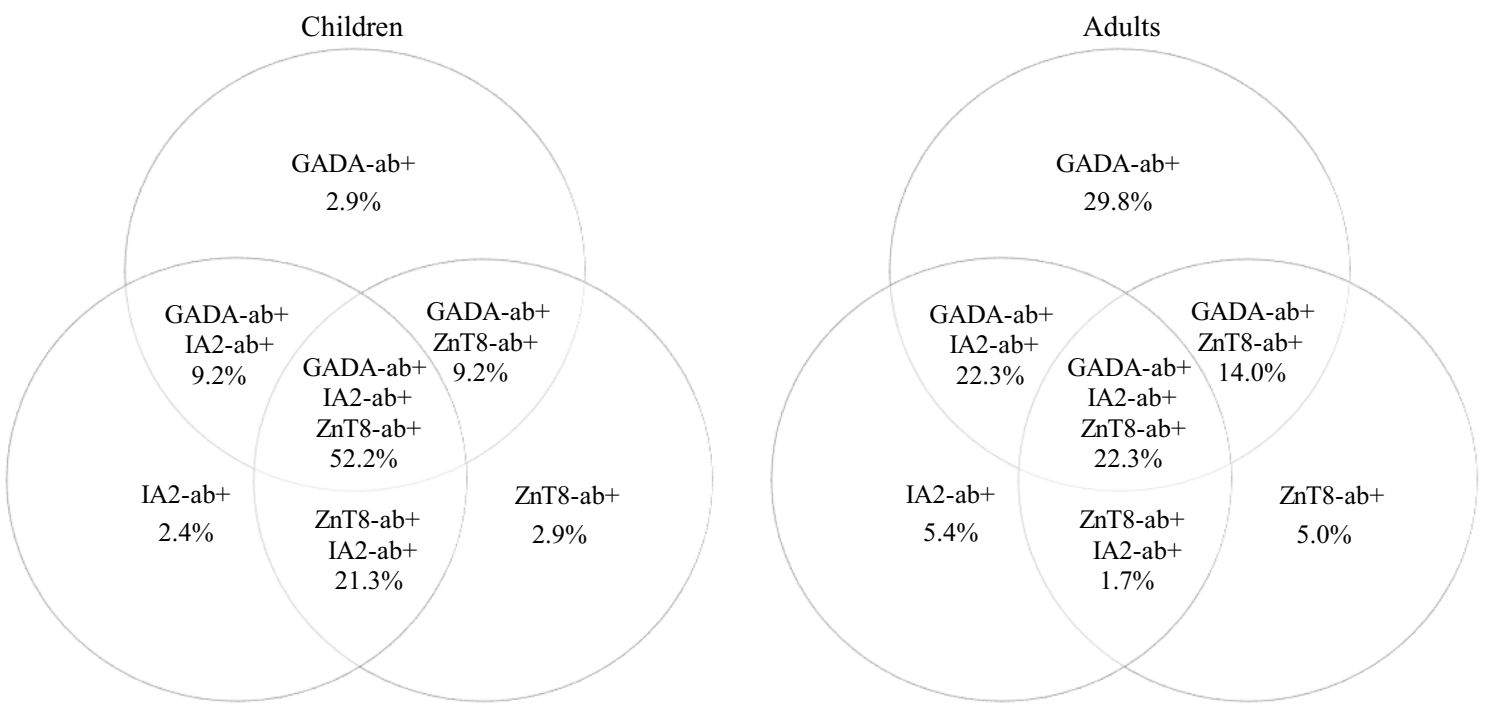

Fig. 2 Venn diagram of autoantibody combinations in autoantibody-positive children and adults at T1DM onset

Table 2 Characteristic of patients with and without DKA at type 1 diabetes onset

\begin{tabular}{llll}
\hline Variable & DKA $(n=78)$ & Without DKA $(n=289)$ & $p$ value \\
\hline Age (year) [IQR] & $9(5-14)$ & $16(9-32)$ & $p<0.001$ \\
ZnT8-ab (\%) & 79.4 & 57.7 & $p=0.002$ \\
ZnT8-ab titer (U/ml) & $250.1(35.6-524.5)$ & $110.5(3.7-524.5)$ & $p=0.031$ \\
IA2-ab (\%) & 78.2 & 61.2 & $p=0.008$ \\
IA2-ab titer (U/ml) & $6.0(2.5-24.2)$ & $11.0(2.7-24.4)$ & NS \\
GAD-ab (\%) & 62.8 & 72.6 & NS \\
GAD-ab titer (U/ml) & $4.7(0.4-34.5)$ & $9.9(2.2-105.4)$ & $p=0.004$ \\
\hline
\end{tabular}

Data are expressed as medians; unless otherwise stated

$I Q R$ interquartile range, $N S$ non-significant

\section{Prevalence of autoantibodies in age subgroups}

Children had higher occurrence of all the three of the assessed antibodies (49.5\%), whereas single antibody was rarely detected $(7.8 \%)$. Adults were mostly positive for just 1 or 2 antibodies, and only $18.1 \%$ of them had three autoantibodies. This may suggest different intensity of $\beta$-cell autoimmunity. In subjects with only one autoantibody, isolated GAD-ab were the most common antibody found in adults, while in children the occurrence of any of three assessed antibodies was similar. In those with 2 positive antibodies, the combination of IA2-ZnT8-abs was the most common in children, but rare in adults. In adults, the combination of GAD-IA2-abs and slightly less frequent GAD-ZnT8 abs were observed. Similarly, as reported by Salonen et al., children were mostly positive for multiple antibodies with predominance of paired ZnT8-ab and IA2-ab [23]. Such an observation might reflect a positive relationship between GAD-ab occurrence and mild form of T1DM onset in adults. The majority of Chinese adults with LADA had only one positive autoantibody (85.9\%), and the most detected single autoantibody was GAD-ab (67\%) [24]. However, our analysis was performed on Caucasian ethnic group.

We observed higher prevalence and titer of ZnT8-ab in children (81.1\%) than in adults (34.8\%). Additionally, higher prevalence of IA-2-ab, but surprisingly lower titer of IA$2-a b$ and GAD-ab, was found in children compared to adults. In contrast, adults had mostly GAD-ab, with higher titer of those autoantibodies. Moreover, a lower prevalence of GAD$\mathrm{ab}$ and a more aggressive diabetes onset in younger children might suggest that with an increase in T1DM prevalence not only a clinical phenotype has changed, but also immunophenotype reflected by a change of GAD-ab occurrence with time. Previously, Long et al. [25] reported the rise of IA2-ab and ZnT8-ab prevalence, while GAD-ab and IAA remained stable.

In our study, the prevalence of ZnT8-ab and other autoantibodies in adults was in concordance with that found in previous studies [26-29]. Compared to these findings in Caucasian populations using internationally validated assays, 
other studies had found an increased prevalence of ZnT8-ab in LADA only in patients with a Japanese ethnic background (19\%) or when the cumulative reactivity to alternative ZnT8 constructs was considered [7, 30]. In children, we found more than $80 \%$ positivity for ZnT8-ab and IA2-ab, but less for GAD-ab. This proportion is slightly different from data reported by the Finnish Pediatric Diabetes Register, where children had mostly IA2-ab (76\%), followed by GAD-ab (67\%) and ZnT8-ab (62.7\%) [23]. Głowińska-Olszewska et al. [31] noticed high prevalence of ZnT8-ab (72\%) in Polish children with T1DM duration shorter than 5 years. This finding demonstrates that ZnT8-ab could identify heterogeneity in the age of diabetes onset and are useful markers of childhood onset T1DM. In the Italian multicenter study, the same sensitivity of ZnT8-ab and GAD-ab at diabetes diagnosis was observed in children [32]. Moreover, ZnT8-abpositive individuals were younger, while GAD-ab positive older at diagnosis [31]. Those observations justify introduction of the three screen assay which detects ZnT8-ab, IA2-ab and GAD-ab, as a useful first steep screening of the $\beta$-cell autoimmunity in children [33].

\section{The diagnostic value of $\mathrm{ZnT}$-ab}

There is a question about the diagnostic value of ZnT8-ab in adults. Mainly, if highest prevalence of ZnT8-ab was observed in children from 3 years old up to late adolescence, a clear tendency for gradual decline in ZnT8-ab was demonstrated thereafter. However, ZnT8-ab are highly $\beta$-cells specific, exclusively expressed in insulin containing secretory granules of $\beta$-cells, and it is a target of the autoimmune process [20,34]. This high specificity emphasizes a diagnostic value of ZnT8-ab among adults mainly in patients with low-titer or absent GAD-ab or in acute onset of T1DM [26]. Particularly, we showed that addition of ZnT8-ab to GAD$\mathrm{ab}$ and IA-2-ab measurements could improve the diagnostic accuracy of autoimmune diabetes in adults, but should be obligatory in children.

\section{Predictive role of autoantibodies in T1DM}

In children, an aggressive autoimmune process may result in overt disease within a few months after the appearance of autoantibodies, whereas in older subjects the preclinical phase may continue for several years. The majority of children had IAA and GAD-ab as their first autoantibodies detectable in preclinical phase [35]. We demonstrated that children had very low titer of GAD-ab compared to adults. Based on our results, it might be also hypothesized that the level of GAD-ab may drop significantly at diagnosis. While, in adults, autoantibodies persisting long after diagnosis are usually high-titer GAD-ab, and much less frequently ZnT8ab or IA2-ab [36]. Similarly, Sosenko et al. [37] indicated that in children GAD-ab titer tend to decline at diagnosis. ZnT8-ab positivity identified subjects at a higher risk of symptomatic diabetes. ZnT8 is located within $\beta$-cells secretory granules, and ZnT8-ab expression may not occur until there is enough $\beta$-cells damage to make this autoantigen perceptible for the immune system. Overall, the number and titer of autoantibodies decline after progression to T1DM. The type of persistent antibodies can be influenced by age at onset and could add information about clinical phenotypes. Perhaps, ZnT8-ab and GAD-ab might be considered as predictive markers for T1DM development, if they were measured in prediabetes phase. It seems that GAD-ab are more useful in such a situation, because they are positive prior to a mild autoimmune diabetes onset. However, in children with potentially short prediabetes phase, the predictive value of ZnT8-ab is not excluded. As, ZnT8-ab might be one of the key players in mediating both insulin secretion and $\beta$-cells mass in T1DM [38].

\section{Relationship between DKA and autoantibodies pattern}

We confirmed that children are at greater risk of DKA at T1DM diagnosis. Yet, factors involved in DKA development and reasons why children are more prone to be diagnosed with DKA are unclear. DKA is either a consequence of delayed diagnosis, or it reflects intensity of destruction of $\beta$-cells. Nevertheless, it is proved that the occurrence of DKA has impact on long-term clinical course, probably through some deleterious influence on lower residual $\beta$-cell function and frequency of remission [39-42]. In our study, subjects with DKA were more likely to be positive for ZnT8$\mathrm{ab}$ and IA-2-ab, but their titer of IA-2-ab and GAD-ab were lower. However, we found a correlation between the number of positive antibodies and the severity of DKA. Furthermore, ZnT8-ab positivity was associated with a higher risk of DKA independent of age and the number of autoantibodies. The association of ZnT8-ab and DKA has been analyzed before with conflicting results. Salonen et al. described that DKA at diagnosis in young children was less common among ZnT8ab-positive subjects. In concordance with previous studies, ZnT8-ab was uncommon among children with the age of diabetes onset below 5 years. There was a steady increase in proportion of ZnT8-ab positivity in patients older than 5 years and a gradual decrease after 10 years of age [23]. Another study in Turkish children showed a high ZnT8-ab prevalence at T1DM onset (58\%), but this was not associated with DKA [43]. However, our results are consistent with those in the Childhood Diabetes in Finland Study Group, in which positivity for ZnT8-ab was associated with older age and more frequent DKA in children at diagnosis as well as lower serum C-peptide concentrations and higher insulin doses over time compared to ZnT8-ab-negative peers [44]. 
Likewise, Kawasaki et al. [45] have shown that about twothirds of adult-onset patients had the slow onset form, and some $20 \%$ present DKA at diagnosis. ZnT8-ab was identified in 58\% patients with acute-onset and in $20 \%$ with slow-onset T1DM among the Japanese.

Overall in our study, ZnT8-ab, IA2-ab and GAD-ab were found in the majority of children, while GAD-ab was the main antibody presented in adults at diagnosis. ZnT8-ab emerged as an important marker for DKA at T1DM onset.

\section{Conclusions}

Children are more prone to develop DKA, and they have lower concentrations of C-peptide at T1DM onset. Consequently, children feature worse residual $\beta$-cell function at diagnosis, which may negatively affect the prognosis of subsequent partial remission. Children and adults demonstrate distinct autoantibody patterns at the autoimmune diabetes diagnosis. ZnT8-ab and IA2-ab are more prevalent in children, while adults often display higher titer of GAD-ab. Our findings suggest that ZnT8-ab are associated with a more acute onset of T1DM due to a more aggressive autoimmune attack.

Authors' contributions EN, BS, MF and PF collected data from pediatric population, and ARF, SP DZZ obtained data from adult populations. All authors contributed substantially to the conception and design of the study, data collection and/or analysis, and writing or revising the manuscript and approved the final version of the manuscript to be published. EN and ARF are the guarantors of this work and, as such, had full access to all the data in the study and take responsibility for the integrity of the data and the accuracy of the data analysis.

\section{Compliance with ethical standards}

Conflict of interest The authors declare that they have no conflict of interest.

Ethical standard statement All procedures followed were in accordance with the ethical standards of the responsible committee on human experimentation (institutional and national) and with the Helsinki Declaration of 1975, as revised in 2008 (5).

Informed consent All participants or their caregivers provided written informed consent before the enrollment in the study.

Open Access This article is distributed under the terms of the Creative Commons Attribution 4.0 International License (http://creativecomm ons.org/licenses/by/4.0/), which permits unrestricted use, distribution, and reproduction in any medium, provided you give appropriate credit to the original author(s) and the source, provide a link to the Creative Commons license, and indicate if changes were made.

\section{References}

1. American Diabetes Association (2015) Classification and diagnosis of diabetes. Diabetes Care 38:S8-S16

2. Bluestone JA, Herold K, Eisenbarth G (2010) Genetics, pathogenesis and clinical interventions in type 1 diabetes. Nature 464:1293-1300

3. Barker JM (2006) Clinical review: type 1 diabetes-associated autoimmunity: natural history, genetic associations, and screening. J Clin Endocrinol Metab 91:1210-1217

4. Atkinson MA, Eisenbarth GS (2001) Type 1 diabetes: new perspectives on disease pathogenesis and treatment. Lancet 358:221-229

5. Eisenbarth GS (1986) Type I diabetes mellitus. A chronic autoimmune disease. N Engl J Med 314:1360-1368

6. Chmiel R, Beyerlein A, Knopff A et al (2015) Early infant feeding and risk of developing islet autoimmunity and type 1 diabetes. Acta Diabetol 52:621-624

7. Trabucchi A, Faccinetti NI, Guerra LL et al (2012) Detection and characterization of ZnT8 autoantibodies could help to screen latent autoimmune diabetes in adult-onset patients with type 2 phenotype. Autoimmunity 45:137-142

8. Wolfsdorf JI, Allgrove J, Craig ME et al (2014) International society for pediatric and adolescent diabetes. ISPAD clinical practice consensus guidelines 2014: diabetic ketoacidosis and hyperglycemic hyperosmolar state. Pediatr Diabetes 15(20):154-179

9. Notkins AL, Lernmark A (2001) Autoimmune type 1 diabetes: resolved and unresolved issues. J Clin Invest 108:1247-1252

10. Sabbah E, Savola K, Kulmala P et al (1999) Diabetes-associated autoantibodies in relation to clinical characteristics and natural course in children with newly diagnosed type 1 diabetes. The Childhood Diabetes In Finland Study Group. J Clin Endocrinol Metab 84:1534-1539

11. Winter WE, Harris N, Schatz D (2002) Type 1 diabetes islet autoantibody markers. Diabetes Technol Ther 4:817-839

12. Vardi P, Ziegler AG, Mathews JH et al (1988) Concentration of insulin autoantibodies at onset of type I diabetes. Inverse loglinear correlation with age. Diabetes Care 11:736-739

13. Ziegler AG, Hummel M, Schenker M, Bonifacio E (1999) Autoantibody appearance and risk for development of childhood diabetes in offspring of parents with type 1 diabetes: the 2-year analysis of the German BABYDIAB Study. Diabetes 48:460-468

14. Genovese S, Bonfanti R, Bazzigaluppi E et al (1996) Association of IA-2 autoantibodies with HLA DR4 phenotypes in IDDM. Diabetologia 39:1223-1226

15. Gorus FK, Goubert P, Semakula C et al (1997) IA-2-autoantibodies complement GAD65-autoantibodies in new-onset IDDM patients and help predict impending diabetes in their siblings. The Belgian Diabetes Registry. Diabetologia 40:95-99

16. Gottlieb DI, Chang YC, Schwob JE (1986) Monoclonal antibodies to glutamic acid decarboxylase. Proc Natl Acad Sci USA 83:8808-8812

17. Lendrum R, Walker G, Cudworth AG, Woodrow JC, Gamble DR (1976) HLA-linked genes and islet-cell antibodies in diabetes mellitus. Br Med J 1:1565-1567

18. Velluzzi F, Secci G, Sepe V et al (2016) Prediction of type 1 diabetes in Sardinian schoolchildren using islet cell autoantibodies: 10-year follow-up of the Sardinian schoolchildren type 1 diabetes prediction study. Acta Diabetol 53:73-79

19. Kohler M, Beyerlein A, Vehik K et al (2017) Joint modeling of longitudinal autoantibody patterns and progression to type 1 diabetes: results from the TEDDY study. Acta Diabetol 11:1009-1017 
20. Wenzlau JM, Frisch LM, Gardner TJ et al (2009) Novel antigens in type 1 diabetes: the importance of ZnT8. Curr Diab Rep 9:105-112

21. Wenzlau JM, Juhl K, Yu L et al (2007) The cation efflux transporter ZnT8 (Slc30A8) is a major autoantigen in human type 1 diabetes. Proc Natl Acad Sci USA 104:17040-17045

22. Kawasaki E, Uga M, Nakamura K et al (2008) Association between anti-ZnT8 autoantibody specificities and SLC30A8 Arg325Trp variant in Japanese patients with type 1 diabetes. Diabetologia 51:2299-2302

23. Salonen KM, Ryhanen S, Harkonen T et al (2013) Autoantibodies against zinc transporter 8 are related to age, metabolic state and HLA DR genotype in children with newly diagnosed type 1 diabetes. Diabetes Metab Res Rev 29:646-654

24. Xiang Y, Huang G, Shan Z et al (2015) Glutamic acid decarboxylase autoantibodies are dominant but insufficient to identify most Chinese with adult-onset non-insulin requiring autoimmune diabetes: LADA China study 5. Acta Diabetol 52:1121-1127

25. Long AE, Gillespie KM, Rokni S et al (2012) Rising incidence of type 1 diabetes is associated with altered immunophenotype at diagnosis. Diabetes 61:683-686

26. Hussein H, Ibrahim F, Sobngwi E et al (2017) Zinc transporter 8 autoantibodies assessment in daily practice. Clin Biochem 50:94-96

27. Wenzlau JM, Liu Y, Yu L et al (2008) A common nonsynonymous single nucleotide polymorphism in the SLC30A8 gene determines ZnT8 autoantibody specificity in type 1 diabetes. Diabetes 57:2693-2697

28. Wenzlau JM, Walter M, Gardner TJ et al (2010) Kinetics of the post-onset decline in zinc transporter 8 autoantibodies in type 1 diabetic human subjects. J Clin Endocrinol Metab 95:4712-4719

29. Lampasona V, Petrone A, Tiberti C et al (2019) Zinc transporter 8 antibodies complement GAD and IA-2 antibodies in the identification and characterization of adult-onset autoimmune diabetes: Non Insulin Requiring Autoimmune Diabetes (NIRAD) 4. Diabetes Care 33:104-108

30. Kawasaki E, Nakamura K, Kuriya G et al (2010) Autoantibodies to insulin, insulinoma-associated antigen-2, and zinc transporter 8 improve the prediction of early insulin requirement in adult-onset autoimmune diabetes. J Clin Endocrinol Metab 95:707-713

31. Glowinska-Olszewska B, Michalak J, Luczynski W et al (2016) Organ-specific autoimmunity in relation to clinical characteristics in children with long-lasting type 1 diabetes. J Pediatr Endocrinol Metab 29:647-656

32. Fabris M, Zago S, Liguori M et al (2015) Anti-zinc transporter protein 8 autoantibodies significantly improve the diagnostic approach to type 1 diabetes: an Italian multicentre study on paediatric patients. Auto Immun Highlights 6:17-22
33. Ziegler AG, Haupt F, Scholz M et al (2016) 3 Screen ELISA for high-throughput detection of beta cell autoantibodies in capillary blood. Diabetes Technol Ther 18:687-693

34. Xu K, Zha M, Wu X et al (2011) Association between rs13266634 $\mathrm{C} / \mathrm{T}$ polymorphisms of solute carrier family 30 member 8 (SLC30A8) and type 2 diabetes, impaired glucose tolerance, type 1 diabetes - a meta-analysis. Diabetes Res Clin Pract 91:195-202

35. Knip M, Siljander H, Ilonen J, Simell O, Veijola R (2016) Role of humoral beta-cell autoimmunity in type 1 diabetes. Pediatr Diabetes $17(22): 17-24$

36. Lampasona V, Liberati D (2016) Islet autoantibodies. Curr Diab Rep 16:53

37. Sosenko JM, Skyler JS, Palmer JP et al (2011) Diabetes Prevention T-T\&Type 1 Diabetes TrialNet Study G. A longitudinal study of GAD65 and ICA512 autoantibodies during the progression to type 1 diabetes in Diabetes Prevention Trial-Type 1 (DPT-1) participants. Diabetes Care 34:2435-2437

38. Dang M, Rockell J, Wagner R et al (2011) Human type 1 diabetes is associated with $\mathrm{T}$ cell autoimmunity to zinc transporter 8 . $\mathrm{J}$ Immunol 186:6056-6063

39. Fernandez Castaner M, Montana E, Camps I et al (1996) Ketoacidosis at diagnosis is predictive of lower residual beta-cell function and poor metabolic control in type 1 diabetes. Diabetes Metab 22:349-355

40. Bowden SA, Duck MM, Hoffman RP (2008) Young children $(<5 \mathrm{yr})$ and adolescents $(>12 \mathrm{yr})$ with type 1 diabetes mellitus have low rate of partial remission: diabetic ketoacidosis is an important risk factor. Pediatr Diabetes 9:197-201

41. Neu A, Ehehalt S, Willasch A et al (2001) Varying clinical presentations at onset of type 1 diabetes mellitus in children-epidemiological evidence for different subtypes of the disease? Pediatr Diabetes 2:147-153

42. Abdul-Rasoul M, Habib H, Al-Khouly M (2006) 'The honeymoon phase' in children with type 1 diabetes mellitus: frequency, duration, and influential factors. Pediatr Diabetes 7:101-107

43. Elmaogullari S, Ucakturk SA, Elbeg S et al (2017) Prevalence of ZnT8 antibody in Turkish children and adolescents with new onset type 1 diabetes. J Clin Res Pediatr Endocrinol. https://doi. org/10.4274/jcrpe.5020 (Epub ahead of print)

44. Juusola M, Parkkola A, Harkonen T et al (2016) Positivity for zinc transporter 8 autoantibodies at diagnosis is subsequently associated with reduced beta-cell function and higher exogenous insulin requirement in children and adolescents with type 1 diabetes. Diabetes Care 39:118-121

45. Kawasaki E (2012) ZnT8 and type 1 diabetes. Endocr J 59:531-537

\section{Affiliations}

\section{Elżbieta Niechciał ${ }^{1}$ (1) Anita Rogowicz-Frontczak ${ }^{2} \cdot$ Stanisław Piłaciński ${ }^{2} \cdot$ Marta Fichna $^{3,4} \cdot$ Bogda Skowrońska $^{1}$. Piotr Fichna' ${ }^{1}$. Dorota Zozulińska-Ziółkiewicz ${ }^{2}$}

1 Department of Pediatric Diabetes and Obesity, Poznan University of Medical Sciences, Szpitalna Street 27/33, 60-572 Poznan, Poland

2 Department of Internal Medicine and Diabetology, Poznan University of Medical Sciences, Mickiewicza Street 2, 60-101 Poznan, Poland
3 Department of Endocrinology, Metabolism and Internal Medicine, Poznan University of Medical Sciences, Przybyszewskiego Street 49, 60-101 Poznan, Poland

4 Institute of Human Genetics, Polish Academy of Sciences, Strzeszyńska Street 32, 60-479 Poznan, Poland 